\title{
Alectinib Together with Intracranial Therapies Improved Survival Outcomes in Untreated ALK-Positive Patients with Non-Small-Cell Lung Cancer and Symptomatic and Synchronic Brain Metastases: A Retrospective Study
}

\section{Qiang Yin \\ Peng Li \\ Peng Wang \\ Zhen Zhang \\ Qun Liu \\ Zengfeng Sun \\ Wenliang Li \\ Li Ma \\ Xiaoguang Wang}

Department of Neurosurgery, Tianjin Medical University Cancer Institute \& Hospital, National Clinical Research

Center for Cancer, Key Laboratory of Cancer Prevention and Therapy, Tianjin's Clinical Research Center for Cancer, Tianjin, 300060, People's Republic of China
Correspondence: Xiaoguang Wang; Li Ma Tel +8622 233401232061 ;

$+8622233401232062$

Fax +862223359337

Email tchwangxg@I63.com; LMa7@tmu. edu.cn
Purpose: The performance of alectinib and crizotinib in untreated anaplastic lymphoma kinase (ALK)-positive patients with non-small-cell lung cancer (NSCLC) and symptomatic and synchronic brain metastases is largely unknown. This retrospective study assessed the effectiveness of alectinib and crizotinib, together with intracranial therapies in a cohort of these patients.

Patients and Methods: This study included 34 previously untreated ALK-positive NSCLC patients with three or fewer intracranial metastases. Of these patients, 13 received oral alectinib $600 \mathrm{mg}$ twice daily, and 21 received oral crizotinib $250 \mathrm{mg}$ twice daily, until progressive disease, unacceptable toxicity, or death. All intracranial metastases were treated with craniotomy, CyberKnife, or both.

Results: Median overall progression-free survival (PFS) was 32.8 months (95\% CI 24.4 41.2 months) in patients treated with alectinib and 8.0 months (95\% CI 7.3-8.7 months) in patients treated with crizotinib. Median PFS of brain lesions was not yet reached with alectinib (95\% CI 30.1 months-not estimated) and was 8.5 months (95\% CI 7.2-12.3 months) with crizotinib. Median PFS of lung lesions was 38.5 months (95\% CI 27.5-49.5 months) with alectinib and 9.2 months (95\% CI 7.4-11.0 months) with crizotinib. Median overall survival was not yet reached with alectinib (95\% CI 31.0 months-not estimated) and 30.3 months (95\% CI 27.3-37.1 months) with crizotinib.

Conclusion: Compared with crizotinib, alectinib showed superior efficacy and lower toxicity in the treatment of ALK-positive patients with NSCLC and symptomatic and synchronic brain metastases. The inclusion of intracranial therapies such as craniotomy or CyberKnife further improved the brain PFS and overall survival of these patients.

Keywords: alectinib, crizotinib, non-small-cell lung cancer, brain metastasis, intracranial therapies

\section{Introduction}

Non-small-cell lung cancer (NSCLC) with anaplastic lymphoma kinase (ALK) rearrangement is a distinct subset of lung cancer, with an incidence ranging from $2 \%$ to $7 \%$. ${ }^{1}$ Echinoderm microtubule associated protein-like 4 gene (EML4) is the absolute dominant ALK fusion partner in NSCLC patients. The EML4-ALK fusion results in the constitutive activation of a pro-proliferative cascade. ${ }^{2}$ These ALK- 
positive tumors tend to have a more aggressive phenotype with multiple metastatic sites at diagnosis compared with other NSCLC types. In particular, patients with ALKpositive NSCLC are usually at higher risk for developing brain metastases, with and the central nervous system (CNS) being the most common site of disease progression. ${ }^{3,4}$ At the time of diagnosis, up to $35 \%$ of these patients had brain metastases, with the rates at 2 and 3 years being $45.5 \%$ and $58.4 \%$, respectively. ${ }^{5}$

Crizotinib is a first-generation ALK inhibitor that showed greater efficacy than chemotherapy in ALK-positive NSCLC patients in the PROFILE 1014 trial. $^{6}$ Subsequent evaluation found that a significant proportion of crizotinib-treated patients developed CNS metastases, probably because the blood-brain barrier is relatively impermeable to crizotinib. Alectinib is a second-generation and highly CNS-active ALK inhibitor that was shown to be highly effective in several Phase III clinical trials. ${ }^{3,7,8}$ For example, the ALEX trial (NCT02075840), which enrolled 303 untreated ALK-positive patients with stage IIIB-IV NSCLC, found that investigator-assessed mature progression-free survival (PFS) was significantly longer in patients treated with alectinib than with crizotinib [hazard ratio (HR) 0.43, 95\% confidence interval (CI) 0.32-0.58]; that median PFS was 34.8 months with alectinib versus 10.9 months with crizotinib; and that median overall survival (OS) was not reached with alectinib versus 57.4 months with crizotinib (stratified HR $0.67,95 \%$ CI $0.46-$ 0.98, $P=0.0376) .{ }^{9}$ The PFS benefit of alectinib was lower but maintained in patients with baseline CNS metastases, with the median PFS being 25.4 months (95\% CI 9.2 months-not estimated) with alectinib and 7.4 months (95\% CI 6.6-9.6 months) with crizotinib (HR 0.37, 95\% CI $0.23-0.58)]$.

However, the proportion of ALK-positive NSCLC patients with baseline CNS metastases was relatively low in the ALEX and other similar phase III clinical trials, with all of these patients having asymptomatic CNS metastases and the occurrence of symptomatic CNS progression being an exclusion criterion. Although wholebrain radiotherapy was allowed in some patients with baseline CNS metastases in the ALEX trial, more advanced intracranial therapies, such as craniotomy or CyberKnife, were not considered or were present in too few patients. These local therapies in terminal cancer patients with three or fewer intracranial metastases were found to effectively alleviate their CNS symptoms and significantly improved their quality of life and overall survival. ${ }^{10,11}$ In addition, local intracranial therapies such as craniotomy or CyberKnife could effectively increase the permeability of the blood-brain barrier and improve the CNS activities of antitumor drugs. ${ }^{12-15}$

To date, the comparative efficacy of alectinib versus crizotinib together with local intracranial therapies in untreated ALK-positive NSCLC patients with symptomatic and synchronic brain metastasis remains largely unknown. The present study retrospectively analyzed the efficacies of alectinib and crizotinib plus intracranial therapies in a cohort of these NSCLC patients who had received or were receiving treatment in our department. This study not only complements the knowledge about the efficacy of ALK inhibitors in lung cancer brain metastases but is also a strong demonstration of the importance of multidisciplinary collaboration in extending the survival of patients with advanced brain metastatic tumors.

\section{Patients and Methods}

\section{Study Population}

Thirty-four patients with NSCLC and obvious neurological symptoms who were diagnosed between August 2016 and October 2019 at their first visit were selected from the inpatient archives of the Neurosurgery Department of Tianjin Medical University Cancer Institute and Hospital (TMUCIH). Patients had not received any prior antitumor treatment and had measurable tumor lesions at baseline according to Response Evaluation Criteria in Solid Tumors (RECIST, version 1.1). None had more than three brain metastases, and all were pathologically confirmed as having lung adenocarcinoma by analysis of either primary or metastatic sites. ALK mutations were detected by Ventana immunohistochemistry, fluorescence in-situ hybridization (FISH), or next-generation sequencing (NGS). None had a previous psychiatric history, severe respiratory/cardiovascular/hepatorenal diseases, or other metastases at the time of initial diagnosis. The median age of the 34 patients was 56 years (range, 35-72 years). Nineteen patients were male, and 15 others were female. Additional demographic and clinical characteristics are shown in Table 1.

\section{Treatment Procedures}

Patients with intracranial metastatic lesions of appropriate location and diameter $>3 \mathrm{~cm}$ underwent craniotomy, with the removed tissue samples subjected to histopathological examination and assessment of gene mutations. Before surgery, all patients underwent relevant examinations, 
Table I Baseline Patient Characteristics

\begin{tabular}{|c|c|c|c|c|c|}
\hline \multirow[t]{2}{*}{ Characteristics } & \multicolumn{2}{|l|}{$\begin{array}{l}\text { Alectinib } \\
(n=13)\end{array}$} & \multicolumn{2}{|l|}{$\begin{array}{l}\text { Crizotinib } \\
(n=21)\end{array}$} & \multirow[t]{2}{*}{$P$ value } \\
\hline & No. & $\%$ & No. & $\%$ & \\
\hline Median age, years (range) & $51(35-72)$ & & $56(37-7 I)$ & & 0.595 \\
\hline Gender & & & & & 1.000 \\
\hline Male & 7 & 53.8 & 12 & 57.1 & \\
\hline Female & 6 & 46.2 & 9 & 42.9 & \\
\hline ECOG performance status & & & & & 1.000 \\
\hline 0 & 3 & 23.1 & 5 & 23.8 & \\
\hline $\mathrm{I}-2$ & 10 & 76.9 & 16 & 76.2 & \\
\hline Smoking history & & & & & 1.000 \\
\hline Never smoked & 6 & 46.2 & 10 & 47.6 & \\
\hline Current smoker or Past smoker & 7 & 53.8 & 11 & 52.4 & \\
\hline Maximum diameter of lung tumors & & & & & 0.727 \\
\hline$\leq 3 \mathrm{~cm}$ & 9 & 69.2 & 13 & 61.9 & \\
\hline$>3 \mathrm{~cm}$ & 4 & 30.8 & 8 & 38.1 & \\
\hline Maximum diameter of CNS metastases & & & & & 0.709 \\
\hline$\leq 3 \mathrm{~cm}$ & 5 & 38.5 & 6 & 28.6 & \\
\hline$>3 \mathrm{~cm}$ & 8 & 61.5 & 15 & 71.4 & \\
\hline Intracranial measurable metastases & & & & & 1.000 \\
\hline 1 & 8 & 61.5 & 12 & 57.1 & \\
\hline $2-3$ & 5 & 38.5 & 9 & 42.9 & \\
\hline CNS maximum metastatic foci treatment & & & & & 0.444 \\
\hline Craniotomy & 11 & 84.6 & 15 & 71.4 & \\
\hline CyberKnife & 2 & 15.4 & 6 & 28.6 & \\
\hline
\end{tabular}

Abbreviation: ECOG, Eastern Cooperative Oncology Group.

with routine blood tests assessing liver and kidney function, coagulation function, tumor markers, and cardiopulmonary function, and their general state was carefully evaluated. Patients with small intracranial lesions but no symptoms of high intracranial pressure underwent computed tomography (CT)-guided percutaneous lung tumor puncture, with the tissue samples subjected to histopathological examination and molecular detection.

All unresected intracranial metastases were treated with CyberKnife radiosurgery. Before treatment, all patients underwent $1.2 \mathrm{~mm}$ thick $\mathrm{T} 1$ enhanced magnetic resonance imaging (MRI) and $1.5 \mathrm{~mm}$ thick CT. Tumor volume was defined as the volume of enhanced focus displayed on enhanced MRI or enhanced CT. After the fusion of CT and MRI images, the gross tumor volume (GTV) was delineated. The planned treatment volume was defined as the GTV plus a positioning error of $1.6 \mathrm{~mm}$. The treatment plan was determined using MultiPlan 4.6.0 treatment plan software. During the treatment, all patients were fixed with facial mesh and located by skull tracking.
Patients diagnosed with ALK-positive lung adenocarcinoma were started immediately on ALK inhibitor therapy. Of the 34 patients, $13(38.2 \%)$ received oral alectinib $600 \mathrm{mg}$ twice daily, and $21(61.8 \%)$ received oral crizotinib $250 \mathrm{mg}$ twice daily, until progressive disease, unacceptable toxicity, or death.

\section{Assessments}

The patients were followed up every 4 weeks until Week 12 and every 8 weeks from Week 12 to progression or death. Patients underwent imaging by $\mathrm{CT}$ and/or MRI at baseline and at regular intervals throughout the study. Tumor response was evaluated using RECIST version 1.1; adverse events were graded according to the National Cancer Institute Common Terminology Criteria for Adverse Events, version 4.0, and were classified according to the Medical Dictionary for Regulatory Activities.

The key study outcomes were PFS, OS, objective response rate (ORR) and safety. Overall PFS was defined 
as the interval from the date of initial diagnosis to confirmation of progressive disease (in the lung, brain, or other organs) or death, whichever occurred first; analogously, the PFS of the lung was defined as the interval from the date of initial diagnosis to confirmation of progressive disease in the lung or death, and PFS of the brain was defined as the interval from the date of initial diagnosis to confirmation of progressive disease in the brain or death. OS was measured from the date of initial diagnosis to the date of death or last follow-up. ORR was defined as the percentage of patients with a complete response (CR) or a partial response (PR). Disease control was defined as no obvious increase in the volume of pre-existing lesions or the occurrence of new lesion on imaging films. Accordingly, disease progression was defined as an increase in the volume of pre-existing lesions or the appearance of new lesions.

\section{Study Oversight}

This study was conducted in accordance with the Declaration of Helsinki and Good Clinical Practice guidelines, and approved by the Ethics Committee of Tianjin Medical University Cancer Institute and Hospital. All patients provided written informed consent prior to their inclusion in the study.

\section{Statistical Analysis}

Survival outcomes were evaluated using the Kaplan-Meier method, and differences between groups were compared by Log rank tests. Hazard ratios (HRs) and 95\% confidence intervals (CIs) were estimated using a Cox regression model. Median survival and $95 \%$ CIs when a $50 \%$ event rate was not observed in any group were assessed using the Brookmeyer and Crowley method. Qualitative variables were compared with the chi-squared or Fisher's exact tests, and quantitative variables were compared using Student's $t$-tests.

All reported P-values were two-sided, with P-values $<0.05$ considered statistically significant. Statistical analyses were performed with IBM SPSS Statistics 19 and SAS 9.4 software.

\section{Results}

\section{Patients}

Of the 34 patients with symptomatic and synchronic brain metastases, $13(38.2 \%)$ were treated with oral alectinib $600 \mathrm{mg}$ twice daily, and $21(61.8 \%)$ were treated with oral crizotinib $250 \mathrm{mg}$ twice daily. The baseline characteristics of the patients in the two groups were well balanced (Table 1). Of these 34 patients, 20 (58.8\%) had single intracranial lesions and $14(41.2 \%)$ had two or more intracranial lesions. Eight of these patients were treated intracranially with the CyberKnife after the diagnosis of NSCLC was confirmed by lung aspiration, whereas the other 26 patients were treated with a craniotomy-focused/ CyberKnife-assisted strategy.

At the cutoff date of May 31, 2021, the median followup was 36.1 months in the alectinib group and 29.7 months in the crizotinib group. The median duration of treatment was 31.1 months (range, 23.4-44.0 months) in the alectinib group and 8 months (range, 3.0-12.3 months) in the crizotinib group. Seven patients in the alectinib group and 21 in the crizotinib group had discontinued treatment.

\section{Efficacy}

The primary lung tumors in almost all patients began to respond within 1 month after the start of ALK-TKI treatment. Of the 13 patients in the alectinib group, all (ORR, $100 \%)$ responded to treatment, including $5(38.5 \%)$ with CR and $8(61.5 \%)$ with PR. Of the 21 patients in the crizotinib group, 13 responded (ORR, 61.9\%), all with PR; whereas the other eight patients (38.1\%) achieved stable disease (SD). Because all intracranial metastases were treated efficiently, with craniotomy, CyberKnife, or both, it was difficult to evaluate the response rate of brain metastases to these two ALK inhibitors.

At the cutoff date of May 31, 2021, an event of disease progression (in any organ) or death had occurred in 28 patients in the whole ALK-TKI treatment population (7 of 13 [53.8\%] patients in the alectinib group and 21 of 21 [100\%] patients in the crizotinib group). The overall PFS rate was significantly higher in the alectinib than in the crizotinib group (HR 0.007, 95\% CI 0.000-0.258, $P<0.001$ ); and the median value for overall PFS was longer in the alectinib (32.8 months; 95\% CI 24.4-41.2 months) than in the crizotinib (8.0 months; $95 \%$ CI $7.3-$ 8.7 months) group (Figure 1A).

Of these 34 patients, 17 (50.0\%) died during the study, including $2(15.4 \%)$ of the 13 patients in the alectinib group and $15(71.4 \%)$ of the 21 patients in the crizotinib group. OS rate was significantly higher with alectinib than with crizotinib (HR $0.141,95 \%$ CI $0.032-0.625$, $P=0.003)$; and median OS was not yet reached with alectinib (95\% CI 31.0 months-not estimated), compared with 

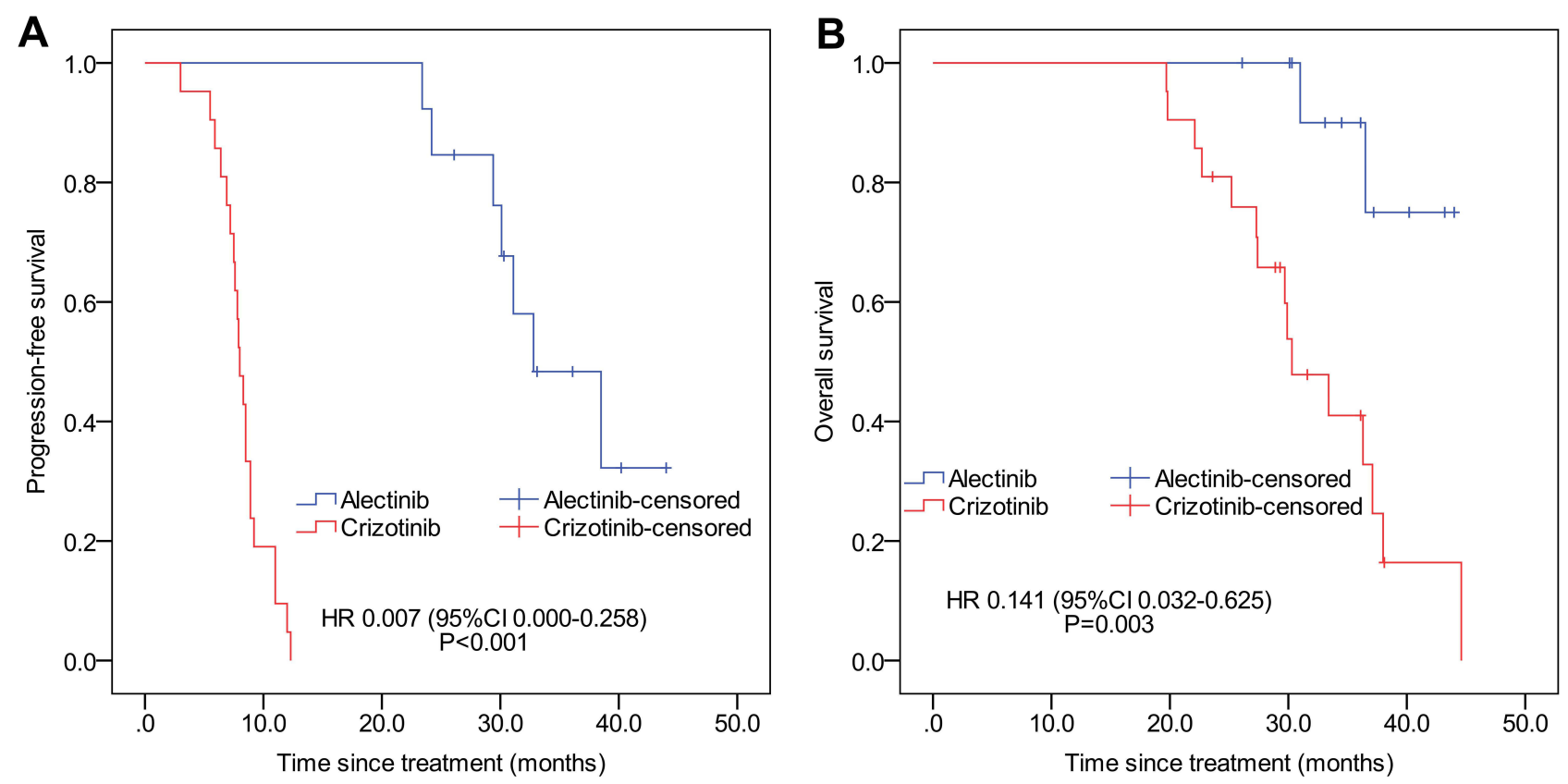

C
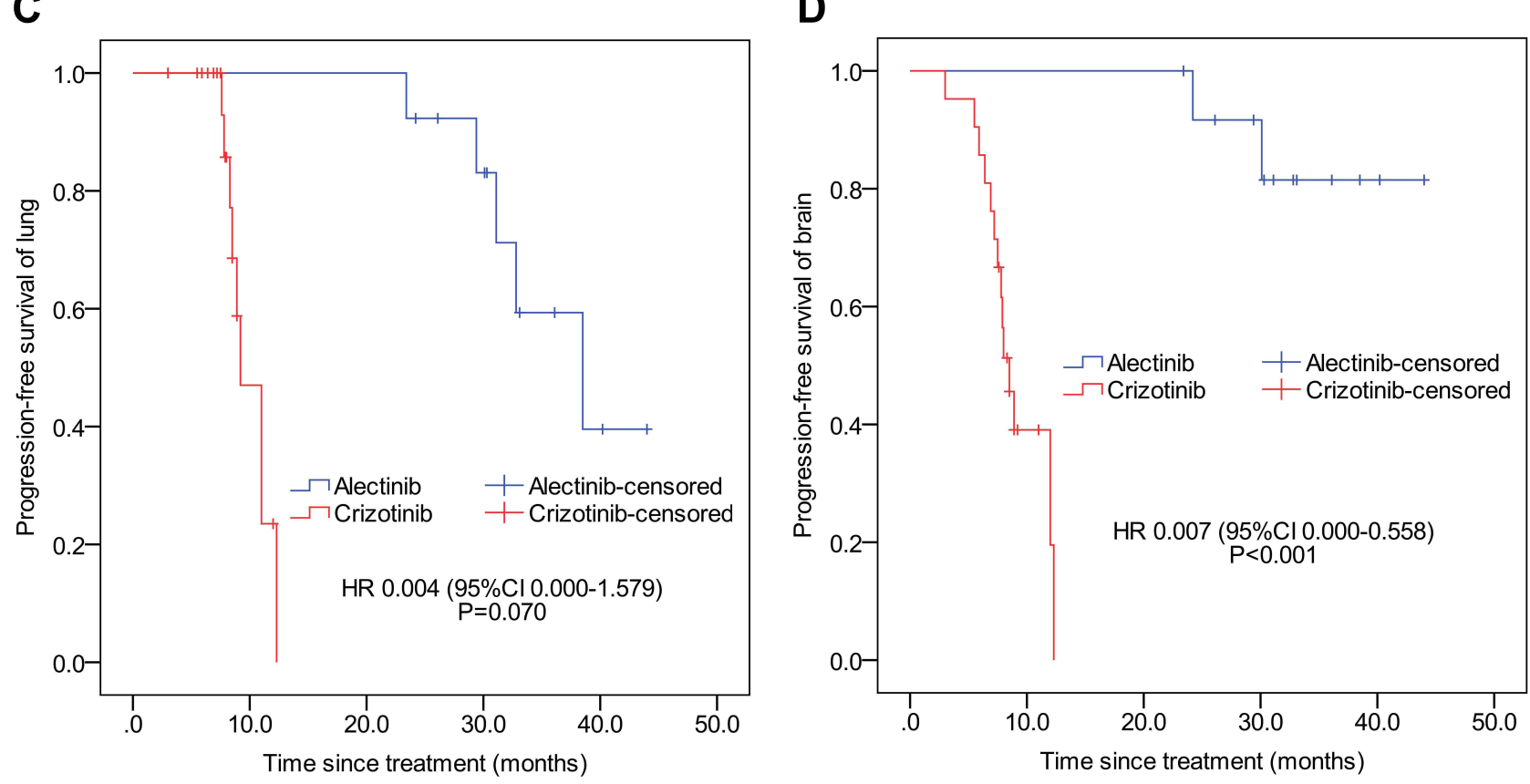

Figure I Kaplan-Meier analyses of survival in patients treated with alectinib and crizotinib. (A) Overall progression-free survival; (B) overall survival; (C) progression-free survival of lung lesions; (D) progression-free survival of brain lesions. P-values were calculated by Log rank tests, and hazard ratios were estimated by Cox regression analysis.

30.3 months (95\% CI 27.3-37.1 months) with crizotinib (Figure 1B).

In addition, the PFS of lung and brain lesions was analyzed. At the cutoff date of May 31, 2021, progression of lung tumors or death had occurred in $14(41.2 \%)$ of the 34 patients, including in five $(38.5 \%)$ of the 13 patients in the alectinib group and nine (42.9\%) of the 21 patients in the crizotinib group). The PFS rate of lung lesions was higher with alectinib than with crizotinib (HR: 0.004, 95\% CI 0.000-1.579), although the difference was not statistically significant $(\mathrm{P}=0.070)$. Median PFS of lung tumors was 38.5 months (95\% CI 27.5-49.5 months) with alectinib and 9.2 months (95\% CI 7.4-11.0 months) with crizotinib (Figure 1C). Progression of brain tumors or death was observed in $16(47.1 \%)$ of the 34 patients, including in two $(15.4 \%)$ of the 13 patients treated with alectinib and 
$14(66.7 \%)$ of the 21 patients treated with crizotinib. The PFS rate of brain lesions was significantly higher with alectinib than with crizotinib (HR 0.007, 95\% CI $0.000-$ $0.558, P<0.001$ ) (Figure 1D); with the median PFS of brain lesions not yet reached with alectinib (95\% CI 30.1 months-not estimated) and 8.5 months (95\% CI 7.2-12.3 months) with crizotinib.

The magnitude of the treatment effect was generally consistent across subgroups (Figure 2), although the $P$ value on Cox regression analysis in some subgroups did not achieve statistical significance due to the small sample size.

\section{Safety}

Adverse events of any grade with higher incidence in patients treated with alectinib than with crizotinib included increased blood creatine phosphokinase (38.4\% vs 38.1\%), sinus bradycardia $(23.1 \%$ vs $19.0 \%)$, vomiting $(15.3 \%$ vs
$14.3 \%)$, nausea $(30.7 \%$ vs $28.6 \%)$, decreased appetite (46.2\% vs $38.1 \%$ ), and rash (23.1\% vs $19.0 \%$ ) (Table 2 ). Adverse events of any grade that were more frequent in patients treated with crizotinib than with alectinib included increased alanine aminotransferase (42.9\% vs $38.4 \%)$, constipation ( $57.1 \%$ vs $46.1 \%$ ), increased blood bilirubin (33.3\% vs $30.7 \%)$, diarrhea (28.6\% vs $23.1 \%)$, decreased white blood cell count $(14.3 \%$ vs $7.0 \%)$, decreased neutrophil count (14.3\% vs $7.0 \%$ ), and blurred vision $(14.3 \%$ vs $7.0 \%)$. Rash (7.6\% vs $4.8 \%$ ) was the only grade III-IV adverse event more frequent in patients treated with alectinib than with crizotinib, whereas grade III-IV adverse events more frequent in patients treated with crizotinib than with alectinib included increased alanine aminotransferase $(23.8 \%$ vs $7.6 \%)$, constipation (38.1\% vs none), increased blood creatine phosphokinase (23.8\% vs $7.6 \%$ ), and increased blood bilirubin (19.0\% vs none). No adverse events resulted in a fatal outcome.

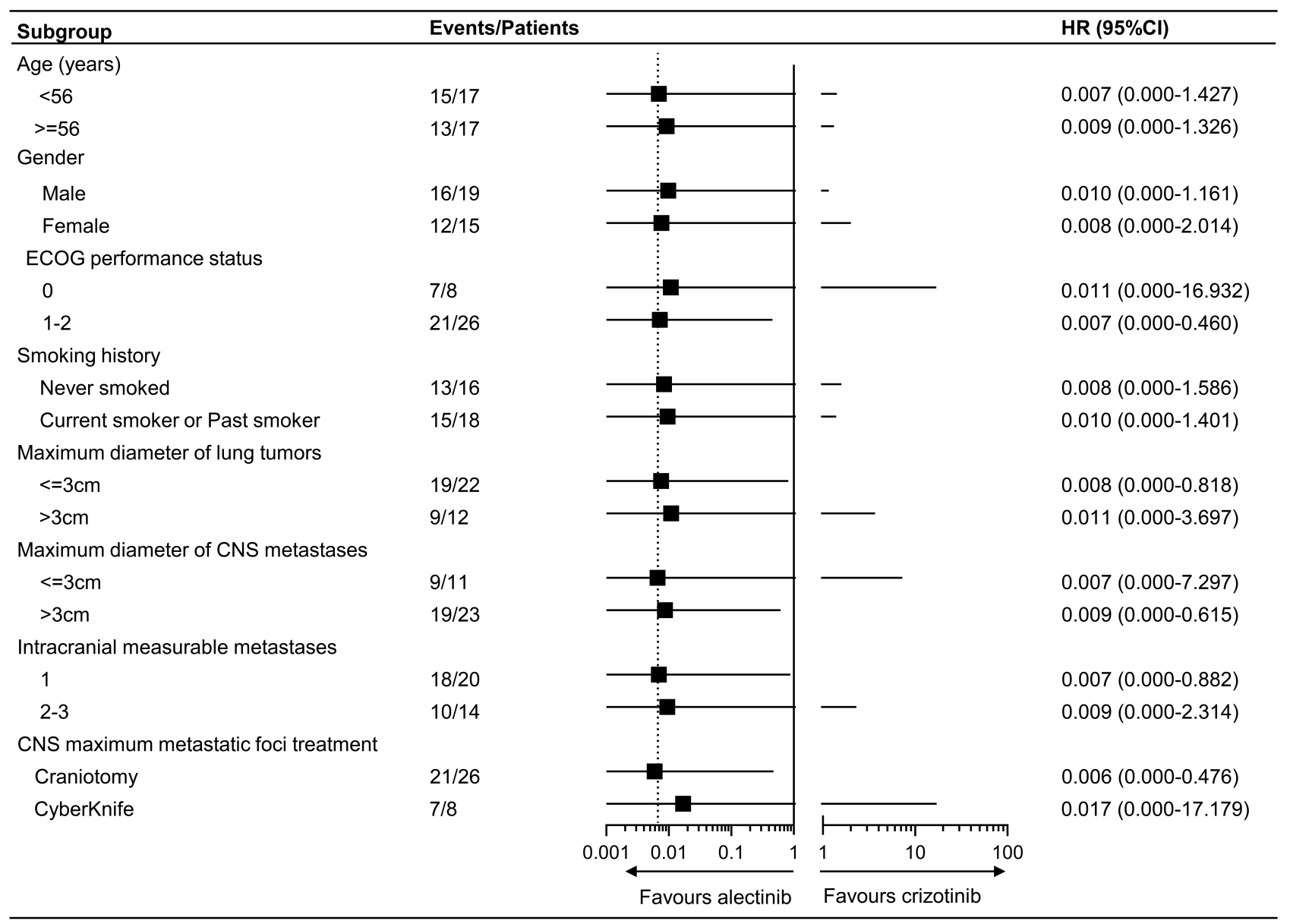

Figure 2 Forest plot of progression-free survival in patient subgroups. Hazard ratio (HR) was estimated by an unstratified Cox model. Abbreviation: ECOG, Eastern Cooperative Oncology Group. 
Table 2 Safety Summary

\begin{tabular}{|l|l|l|l|l|}
\hline \multirow{2}{*}{ Index } & \multicolumn{2}{|c|}{ Any Grade } & \multicolumn{2}{c|}{ Grade III-IV } \\
\cline { 2 - 5 } & Alectinib & Crizotinib & Alectinib & Crizotinib \\
\hline Increased alanine aminotransferase & $5 / 13(38.4 \%)$ & $9 / 21(42.9 \%)$ & $1 / 13(7.6 \%)$ & $5 / 21(23.8 \%)$ \\
Constipation & $6 / 13(46.1 \%)$ & $12 / 21(57.1 \%)$ & $0 / 13(0 \%)$ & $8 / 21(38.1 \%)$ \\
Increased blood creatine phosphokinase & $5 / 13(38.4 \%)$ & $8 / 21(38.1 \%)$ & $1 / 13(7.6 \%)$ & $5 / 21(23.8 \%)$ \\
Increased blood bilirubin & $4 / 13(30.7 \%)$ & $7 / 21(33.3 \%)$ & $0 / 13(0 \%)$ & $4 / 21(19.0 \%)$ \\
Sinus bradycardia & $3 / 13(23.1 \%)$ & $4 / 21(19.0 \%)$ & $0 / 13(0 \%)$ & $0 / 21(0 \%)$ \\
Vomiting & $2 / 13(15.3 \%)$ & $3 / 21(14.3 \%)$ & $0 / 13(0 \%)$ & $0 / 21(0 \%)$ \\
Diarrhea & $3 / 13(23.1 \%)$ & $6 / 21(28.6 \%)$ & $0 / 13(0 \%)$ & $0 / 21(0 \%)$ \\
Nausea & $4 / 13(30.7 \%)$ & $6 / 21(28.6 \%)$ & $0 / 13(0 \%)$ & $0 / 21(0 \%)$ \\
Decreased appetite & $6 / 13(46.2 \%)$ & $8 / 21(38.1 \%)$ & $0 / 13(0 \%)$ & $0 / 21(0 \%)$ \\
Rash & $3 / 13(23.1 \%)$ & $4 / 21(19.0 \%)$ & $1 / 13(7.6 \%)$ & $1 / 21(4.8 \%)$ \\
Decreased white blood cell count & $1 / 13(7.0 \%)$ & $3 / 21(14.3 \%)$ & $0 / 13(0 \%)$ & $0 / 21(0 \%)$ \\
Decreased neutrophil count & $1 / 13(7.0 \%)$ & $3 / 21(14.3 \%)$ & $0 / 13(0 \%)$ & $0 / 21(0 \%)$ \\
Blurred vision & $1 / 13(7.0 \%)$ & $3 / 21(14.3 \%)$ & $0 / 13(0 \%)$ & $0 / 21(0 \%)$ \\
\hline
\end{tabular}

\section{Post-Progression Therapies}

Of the 21 patients in the crizotinib group, eight (38.1\%) were switched to alectinib (600 $\mathrm{mg}$ twice daily) after the first progression; however, seven of these eight patients experienced a second progression within 11 months.

These 7 patients, together with the 13 patients in the crizotinib group who refused alectinib treatment after first progression, received chemotherapy, intracranial CyberKnife or whole-brain radiotherapy as post-progression therapies. Of the seven alectinib-treated patients with disease progression, three received chemotherapy, one underwent intracranial CyberKnife treatment, one received ceritinib, one underwent intracranial CyberKnife treatment plus a higher dose of alectinib, and one received chemotherapy plus alectinib.

The efficacy difference of alectinib as first-line and second-line treatment was compared in the study population. Kaplan-Meier analysis showed that alectinib was far more effective as first-line than as second-line therapy $(P<0.001$, Figure 3$)$. The median PFS was longer in patients who received first-line (32.8 months; 95\% CI 24.4-41.2 months) than second-line (7.8 months; 95\% CI 4.5-11.1 months) alectinib.

\section{Discussion}

Patients with ALK-positive advanced NSCLC are characterized by onset at a young age, a predisposition to brain metastases, and poor prognosis. ${ }^{16,17}$ Approximately 15-35\% of ALK-positive NSCLC patients are initially diagnosed with CNS metastases, with their presence at baseline being prognostic of poor outcomes. ${ }^{18}$
Crizotinib was the first ALK inhibitor to be developed. However, a retrospective study of patients without baseline brain metastases who developed progressive disease after initiation of crizotinib found that $20 \%$ had developed brain metastases; moreover, the ORR of 109 NSCLC patients with baseline brain metastases who were treated with crizotinib was only $18 \% .{ }^{19}$ This lack of efficacy may be related to the relative impermeability of the brain-blood barrier (BBB) to crizotinib. ${ }^{20,21}$

Ceritinib was a second-generation ALK inhibitor. The ASCEND-4 trial, comparing ceritinib and chemotherapy in ALK-positive NSCLC patients with brain metastases, found that the ORRs were $72.7 \%$ and $27.3 \%$, respectively, and that these groups had a median PFS of 10.7 and 6.6 months (HR 0.70; 95\% CI 0.44-1.12), respectively. ${ }^{22}$ Although ceritinib was somewhat effective against intracranial metastases, it had low brain permeability due to the presence of P-glycoprotein in the BBB. ${ }^{23}$ Thus, the concentration of ceritinib in the brain failed to reach the exposure level needed to control the disease. Among ALK-positive NSCLC patients who progressed after ceritinib treatment, $42 \%$ had CNS metastases as the first symptom. ${ }^{22}$

Compared with crizotinib, alectinib had better efficacy and lower toxicity in the treatment of ALK-positive NSCLC. ${ }^{3}$ In addition, alectinib was not a substrate of P-glycoprotein and was able to cross the BBB, with its concentration in cerebrospinal fluid being higher than the concentrations of crizotinib and ceritinib. ${ }^{24}$ The J-ALEX study compared the efficacy and safety of alectinib with crizotinib in Japanese patients with advanced ALK- 


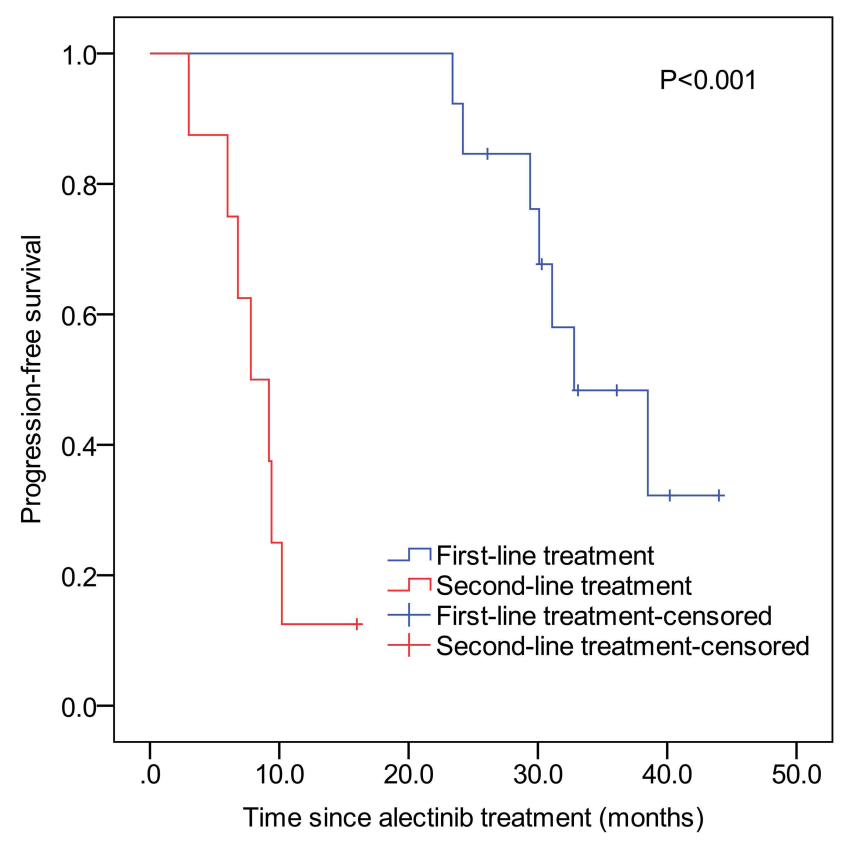

Figure 3 Kaplan-Meier analysis of progression-free survival in patients receiving first-line and second-line alectinib treatment. The $P$ value was calculated by Log rank test.

positive NSCLC, with an independent review committee finding that median PFS was longer in patients treated with alectinib (34.1 months) than with crizotinib (10.2 months). ${ }^{25}$ The ALESIA study also found that the independent review committee-assessed PFS was significantly longer in the alectinib than in the crizotinib group (HR $0.37,0.22-0.61 ; P<0.0001)$, and that CNS ORR in patients with measurable or non-measurable baseline CNS lesions was higher in patients treated with alectinib (32/44, 73\%) than with crizotinib $(5 / 23,22 \%){ }^{8}$ Despite the longer treatment duration with alectinib than with crizotinib, lower percentage of alectinib-treated patients had grade 3-5 or serious adverse events. ${ }^{8,25}$

In contrast to the ALEX, J-ALEX and ALESIA trials, in which the neurological symptoms of ALK-positive NSCLC patients with baseline CNS metastases were all not obvious and the proportion of patients with baseline CNS metastases was very low, the present study assessed ALK-positive NSCLC patients who were initially diagnosed with symptomatic and synchronic brain metastases. Moreover, their intracranial metastases were all treated efficiently, with craniotomy, CyberKnife, or both, prior to starting an ALK inhibitor. Although traumatic brain injury or stereotactic radiosurgery has been reported to disrupt the integrity of the $\mathrm{BBB},{ }^{12-15}$ the efficacy of crizotinib in this study was no greater than that of earlier clinical trials.
Alectinib still remains the first ALK inhibitor of choice for treating NSCLC patients with obvious neurological symptoms at the time of initial diagnosis.

Despite well-established management approaches for brain metastases, with local intracranial therapies as the cornerstone, the initial treatment option for oncogene-driven NSCLC patients with baseline brain metastases remains unclear. For example, Makoto Nishino et al suggested that systemic treatment with a new-generation tyrosine kinase inhibitor (TKI), such as alectinib or osimertinib, was preferred as the initial intervention for these patients, as these new targeted therapies had demonstrated far greater intracranial efficacy than historically favored local therapies. ${ }^{26}$ However, for brain metastatic patients with obvious neurological symptoms, timely and efficient intracranial therapies could rapidly reduce the brain tumor burden and relieve their intracranial symptoms, thus being of great significance to these patients. The ALK-positive NSCLC patients in the present study who underwent craniotomy or CyberKnife treatment followed by an ALK-TKI achieved a better median PFS than patients with baseline brain metastases in the ALEX trial, with median PFS with alectinib of 32.8 and 25.4 months, respectively; and median PFS with crizotinib of 8.0 and 7.4 months, respectively. Although the sample size was relatively small in the present study, these results suggest that, in addition to alectinib, aggressive local therapies such as craniotomy and CyberKnife should be an essential part of the first-line treatment plan for these patients.

Another notable result in the present study was that alectinib was more effective as first-line (median PFS 32.8 months) than as second-line (median PFS 7.8 months) treatment in this patient cohort. The median PFS of alectinib second-line therapy observed in the present study was consistent with the results of the phase III ALUR trial and the Phase II NP28673 and NP28761 studies. In the ALUR study, patients with advanced ALK-positive NSCLC who had progressed after crizotinib treatment had a median independent review committee-assessed PFS of 7.1 months (95\% CI 6.3-10.8 months) with alectinib and 1.6 months (95\% CI 1.3-4.1 months) with chemotherapy. ${ }^{27} \mathrm{~A}$ pooled analysis of two single-arm phase II studies, NP28673 and NP28761, of patients with crizotinib-resistant ALK-positive NSCLC treated with alectinib treatment found that the systemic ORR was $51 \%$ and the median PFS was 8.3 months. ${ }^{28}$ The reduced effectiveness of alectinib as second-line than as first-line therapy may be related to the complex mechanism of ALK inhibitor 
resistance. Almost all patients treated with crizotinib would inevitably relapse and progress, despite its initial effectiveness. Crizotinib resistance has been reported as due, at least in part, to mutations in the ALK kinase domain, alternative bypass pathway activation, and epithelial to mesenchymal transition. ${ }^{29}$ A study in H3122derived ALK TKI-resistant cell lines found that the development in tumors of resistance to a particular ALK TKI would lead to substantial cross-resistance to other ALK TKIs that were not previously present. ${ }^{30}$ This cross-resistance suggests that the efficacy of alectinib is maximized by its use as first-line therapy, especially in NSCLC patients with baseline brain metastases.

This study had several limitations. First, due to its small sample size, the statistical significance of certain results could not be fully demonstrated. Second, because the follow-up time was relatively short, the median PFS of brain lesions and median OS had not yet been reached in the alectinib group. Third, intracranial treatment in this study did not include whole-brain radiotherapy. Wholebrain radiotherapy has been shown to be very important in prolonging survival of patients with lung cancer and brain metastases, especially those with multiple brain metastases. Last, this study did not include a molecular subtype analysis of ALK fusion variants, because of the lack of second-generation sequencing results in some patients and the small sample size.

\section{Conclusion}

In this study, alectinib showed superior efficacy and lower toxicity compared with crizotinib in the treatment of ALKpositive NSCLC patients with symptomatic and synchronic brain metastases, and that alectinib was more effective as first-line than as second-line therapy. In addition to alectinib, aggressive local therapies such as craniotomy and CyberKnife should be an essential part of the firstline treatment plan for these patients.

\section{Acknowledgments}

We thank Dr Guoqiang Chang of Virginia Commonwealth University for his help in language editing. This work was supported by the National Natural Science Foundation of China (81702481) and Young Innovative Talents Training Program of Tianjin Medical University Cancer Institute and Hospital (TMUCIH-2019-1-9).

\section{Disclosure}

The authors report no conflicts of interest in this work.

\section{References}

1. Kwak EL, Bang YJ, Camidge DR, et al. Anaplastic lymphoma kinase inhibition in non-small-cell lung cancer. $N$ Engl J Med. 2010;363 (18):1693-1703. doi:10.1056/NEJMoa1006448

2. Zhang SS, Nagasaka M, Zhu VW, Ou SI. Going beneath the tip of the iceberg. Identifying and understanding EML4-ALK variants and TP53 mutations to optimize treatment of ALK fusion positive (ALK+) NSCLC. Lung Cancer. 2021;158:126-136. doi:10.1016/j. lungcan.2021.06.012

3. Peters S, Camidge DR, Shaw AT, et al. Alectinib versus crizotinib in untreated ALK -positive non-small-cell lung cancer. $N$ Engl J Med. 2017;377(9):829-838. doi:10.1056/NEJMoa1704795

4. Guerin A, Sasane M, Zhang J, et al. Brain metastases in patients with ALK + non-small cell lung cancer: clinical symptoms, treatment patterns and economic burden. J Med Econ. 2015;18(4):312-322. doi:10.3111/13696998.2014.1003644

5. Rangachari D, Yamaguchi N, VanderLaan PA, et al. Brain metastases in patients with EGFR-mutated or ALK-rearranged non-small-cell lung cancers. Lung Cancer. 2015;88(1):108-111. doi:10.1016/j. lungcan.2015.01.020

6. Solomon BJ, Mok T, Kim DW, et al. First-line crizotinib versus chemotherapy in ALK-positive lung cancer. $N$ Engl $J$ Med. 2014;371(23):2167-2177. doi:10.1056/NEJMoa1408440

7. Hida T, Nokihara H, Kondo M, et al. Alectinib versus crizotinib in patients with ALK-positive non-small-cell lung cancer (J-ALEX): an open-label, randomised Phase 3 trial. Lancet. 2017;390(10089):2939. doi:10.1016/S0140-6736(17)30565-2

8. Zhou C, Kim SW, Reungwetwattana T, et al. Alectinib versus crizotinib in untreated Asian patients with anaplastic lymphoma kinasepositive non-small-cell lung cancer (ALESIA): a randomised phase 3 study. Lancet Respir Med. 2019;7(5):437-446. doi:10.1016/S22132600(19)30053-0

9. Mok T, Camidge DR, Gadgeel SM, et al. Updated overall survival and final progression-free survival data for patients with treatmentnaive advanced ALK-positive non-small-cell lung cancer in the ALEX study. Ann Oncol. 2020;31(8):1056-1064. doi:10.1016/j. annonc.2020.04.478

10. Brown PD, Ballman KV, Cerhan JH, et al. Postoperative stereotactic radiosurgery compared with whole brain radiotherapy for resected metastatic brain disease (NCCTG N107C/CEC.3): a multicentre, randomised, controlled, phase 3 trial. Lancet Oncol. 2017;18 (8):1049-1060. doi:10.1016/S1470-2045(17)30441-2

11. Achrol AS, Rennert RC, Anders C, et al. Brain metastases. Nat Rev Dis Primers. 2019;5(1):5. doi:10.1038/s41572-018-0055-y

12. Chodobski A, Zink BJ, Szmydynger-Chodobska J. Blood-brain barrier pathophysiology in traumatic brain injury. Transl Stroke Res. 2011;2(4):492-516. doi:10.1007/s12975-011-0125-x

13. Ho KM, Honeybul S, Yip CB, Silbert BI. Prognostic significance of blood-brain barrier disruption in patients with severe nonpenetrating traumatic brain injury requiring decompressive craniectomy. $\quad J$ Neurosurg. 2014;121(3):674-679. doi:10.3171/2014.6. JNS132838

14. Sulhan S, Lyon KA, Shapiro LA, Huang JH. Neuroinflammation and blood-brain barrier disruption following traumatic brain injury: pathophysiology and potential therapeutic targets. $J$ Neurosci Res. 2020;98(1):19-28. doi:10.1002/jnr.24331

15. Soliman H, Das S, Larson DA, Sahgal A. Stereotactic radiosurgery (SRS) in the modern management of patients with brain metastases. Oncotarget. 2016;7(11):12318-12330. doi:10.18632/onco target.7131

16. Sacher AG, Dahlberg SE, Heng J, Mach S, Janne PA, Oxnard GR. Association between younger age and targetable genomic alterations and prognosis in non-small-cell lung cancer. JAMA Oncol. 2016;2 (3):313-320. doi:10.1001/jamaoncol.2015.4482 
17. Lee JK, Park HS, Kim DW, et al. Comparative analyses of overall survival in patients with anaplastic lymphoma kinase-positive and matched wild-type advanced nonsmall cell lung cancer. Cancer. 2012;118(14):3579-3586. doi:10.1002/cncr.26668

18. Zhang I, Zaorsky NG, Palmer JD, Mehra R, Lu B. Targeting brain metastases in ALK-rearranged non-small-cell lung cancer. Lancet Oncol. 2015;16(13):e510-e521. doi:10.1016/S1470-2045(15)00013-3

19. Metro G, Lunardi G, Floridi P, et al. CSF concentration of crizotinib in two ALK-positive non-small-cell lung cancer patients with CNS metastases deriving clinical benefit from treatment. $J$ Thorac Oncol. 2015;10(5):e26-e27. doi:10.1097/JTO.0000000000000468

20. Kim S, Kim TM, Kim DW, et al. Heterogeneity of genetic changes associated with acquired crizotinib resistance in ALK-rearranged lung cancer. $J$ Thorac Oncol. 2013;8(4):415-422. doi:10.1097/ JTO.0b013e318283dcc0

21. Marsilje TH, Pei W, Chen B, et al. Synthesis, structure-activity relationships, and in vivo efficacy of the novel potent and selective anaplastic lymphoma kinase (ALK) inhibitor 5-chloro-N2-(2-isopropoxy-5-methyl-4-(piperidin-4-yl)phenyl)-N4-(2-(isopropylsulfonyl) phenyl)pyrimidine-2,4-diamine (LDK378) currently in Phase 1 and Phase 2 clinical trials. J Med Chem. 2013;56(14):5675-5690. doi: $10.1021 / \mathrm{jm} 400402 \mathrm{q}$

22. Soria J-C, Tan DSW, Chiari R, et al. First-line ceritinib versus platinum-based chemotherapy in advanced ALK -rearranged nonsmall-cell lung cancer (ASCEND-4): a randomised, open-label, phase 3 study. Lancet. 2017;389(10072):917-929. doi:10.1016/ S0140-6736(17)30123-X

23. Kort A, Sparidans RW, Wagenaar E, Beijnen JH, Schinkel AH. Brain accumulation of the EML4-ALK inhibitor ceritinib is restricted by P-glycoprotein (P-GP/ABCB1) and breast cancer resistance protein (BCRP/ABCG2). Pharmacol Res. 2015;102:200-207. doi:10.1016/j. phrs.2015.09.003
24. Kodama T, Hasegawa M, Takanashi K, Sakurai Y, Kondoh O, Sakamoto H. Antitumor activity of the selective ALK inhibitor alectinib in models of intracranial metastases. Cancer Chemother Pharmacol. 2014;74(5):1023-1028. doi:10.1007/s00280-014-2578-6

25. Nakagawa K, Hida T, Nokihara $H$, et al. Final progression-free survival results from the J-ALEX study of alectinib versus crizotinib in ALK-positive non-small-cell lung cancer. Lung Cancer. 2020;139:195-199. doi:10.1016/j.lungcan.2019.11.025

26. Nishino M, Soejima K, Mitsudomi T. Brain metastases in oncogenedriven non-small cell lung cancer. Transl Lung Cancer Res. 2019;8 (Suppl 3):S298-S307. doi:10.21037/tlcr.2019.05.15

27. Novello S, Mazieres J, Oh IJ, et al. Alectinib versus chemotherapy in crizotinib-pretreated anaplastic lymphoma kinase (ALK)-positive non-small-cell lung cancer: results from the phase III ALUR study. Ann Oncol. 2018;29(6):1409-1416. doi:10.1093/annonc/mdy121

28. Yang JC, Ou SI, De Petris L, et al. Pooled systemic efficacy and safety data from the pivotal Phase II studies (NP28673 and NP28761) of alectinib in ALK-positive non-small cell lung cancer. $J$ Thorac Oncol. 2017;12(10):1552-1560. doi:10.1016/j.jtho.2017.06.070

29. Dong X, Fernandez-Salas E, Li E, Wang S. Elucidation of resistance mechanisms to second-generation ALK inhibitors alectinib and ceritinib in non-small cell lung cancer cells. Neoplasia. 2016;18(3):162171. doi:10.1016/j.neo.2016.02.001

30. Dhawan A, Nichol D, Kinose F, et al. Collateral sensitivity networks reveal evolutionary instability and novel treatment strategies in ALK mutated non-small cell lung cancer. Sci Rep. 2017;7(1):1232.
OncoTargets and Therapy

\section{Publish your work in this journal}

OncoTargets and Therapy is an international, peer-reviewed, open access journal focusing on the pathological basis of all cancers, potential targets for therapy and treatment protocols employed to improve the management of cancer patients. The journal also focuses on the impact of management programs and new therapeutic

\section{Dovepress}

agents and protocols on patient perspectives such as quality of life, adherence and satisfaction. The manuscript management system is completely online and includes a very quick and fair peer-review system, which is all easy to use. Visit http://www.dovepress.com/ testimonials.php to read real quotes from published authors. 